\title{
Stations \\ de transfert d'énergie en service, en construction oul en projet
}

\section{GROUPES TERNAIRES (caractéristiques de la station)}

Abréviations : F. : Francis / P : Pelton / Ppe : Pompe / Horizontal

\begin{tabular}{|c|c|c|c|c|c|c|c|c|c|c|c|}
\hline \multirow[b]{2}{*}{ ys } & \multirow[b]{2}{*}{ Station } & \multirow[b]{2}{*}{$\begin{array}{l}\text { Nombre } \\
\text { de groupes } \\
\text { et types }\end{array}$} & \multicolumn{2}{|c|}{ Turbine } & \multicolumn{3}{|c|}{ Pompe } & \multirow[b]{2}{*}{$\begin{array}{l}\text { Vitesse } \\
(\mathrm{tr} / \mathrm{mn})\end{array}$} & \multicolumn{2}{|c|}{ Constructeurs } & \multirow[b]{2}{*}{$\begin{array}{c}\text { Année } \\
\text { de mise } \\
\text { en } \\
\text { service }\end{array}$} \\
\hline & & & $\begin{array}{c}\text { Hauteur } \\
\text { de chute } \\
\text { nette } \\
\text { (m) }\end{array}$ & $\begin{array}{c}\text { Puissance } \\
\text { totale } \\
\text { installée } \\
\text { (MW) }\end{array}$ & $\begin{array}{c}\text { Hauteur } \\
\text { totale } \\
\text { d'éléva- } \\
\text { tion } \\
(\mathrm{m}) \\
\end{array}$ & $\begin{array}{l}\text { Débit } \\
\text { total } \\
\left(\mathrm{m}^{3 /} / \mathrm{s}\right) \\
\end{array}$ & $\begin{array}{c}\begin{array}{c}\text { Puissance } \\
\text { totale } \\
\text { absorbee }\end{array} \\
\text { (MW) }\end{array}$ & & $\begin{array}{l}\text { Turbine } \\
\text { et pompe }\end{array}$ & $\begin{array}{l}\text { Alternateur } \\
\text { moteur }\end{array}$ & \\
\hline emagne & Waldshut & $4 \mathrm{FH}$ & 160 & 176 & 158,5 & 40 & 80 & 250 & Voith & Siemens - B.B.C. & 1953 \\
\hline édérale & Witznau & $4 \mathrm{FV}$ & 250 & 220 & 260 & 40 & 128 & 333 & $\begin{array}{c}\text { Escher-Wyss } \\
\text { Voith }\end{array}$ & B.B.C. & 1943 \\
\hline $\begin{array}{l}\text { Groupe du } \\
\text { Schluchsee }\end{array}$ & Haüsern & $4 F V$ & 210 & 140 & 234 & 40 & 104 & 333 & $\begin{array}{c}\text { Escher-Wyss } \\
\text { Voith }\end{array}$ & Siemens - B.B.C. & 1933 \\
\hline \multirow{14}{*}{$\begin{array}{l}\text { Iroupe du } \\
\text { totzenwald }\end{array}$} & Säkingen & $4 \mathrm{FH}$ & 413 & 352 & 413 & 64 & 282 & 600 & $\begin{array}{c}\text { Escher-Wyss } \\
\text { Voith }\end{array}$ & A.E.G. - B.B.C. & 1968 \\
\hline & Hornberg (Wehr) & $4 F V$ & 652 & 992 & 664 & 144 & 1020 & 600 & $\begin{array}{c}\text { Escher-Wyss } \\
\text { Voith }\end{array}$ & Siemens & 1976 \\
\hline & Waldeck I & $4 \mathrm{FH}$ & 296 & 140 & 308 & 24 & 96 & 500 & $\begin{array}{c}\text { Escher-Wyss } \\
\text { Voith }\end{array}$ & & 1932 \\
\hline & Waldeck II & $2 \mathrm{FV}$ & 329 & 440 & 329 & 67 & 460 & 375 & $\begin{array}{c}\text { Escher-Wyss } \\
\text { Voith }\end{array}$ & A.E.G. & 1975 \\
\hline & Waldeck III & $2 F$ & 338 & 239 & 329 & 67 & 220 & 375 & & & projet \\
\hline & Herdecke & $4 \mathrm{FH}$ & 165 & 132 & 165 & 56,2 & 106,8 & & Voith & Siemens & 1930 \\
\hline & Happurg & $4 \mathrm{FH}$ & 208 & 144 & 218 & 51 & 128 & 375 & Voith & Siemens & 1959 \\
\hline & Reisach-Rabenleite & $3 \mathrm{FH}$ & 166 & 104 & 170 & 43,5 & 83 & 333 & Voith & Siemens - A.E.G. & 1955 \\
\hline & Geesthacht I & $3 \mathrm{FH}$ & 76 & 131 & 84 & 100 & 104 & 214 & $\begin{array}{c}\text { Escher-Wyss } \\
\text { Voith }\end{array}$ & Siemens & 1958 \\
\hline & Tanzmühle & $1 \mathrm{FH}$ & 122 & 28 & 139 & 17 & 25 & 375 & Voith & Siemens & 1959 \\
\hline & Leitzachwerk II & $2 \mathrm{FH}$ & 125 & 48 & 125 & 27 & 40 & 428 & Escher-Wyss & Siemens & 1960 \\
\hline & Glems & $2 \mathrm{FH}$ & 293 & 90 & 298 & 20 & 64 & 600 & $\begin{array}{c}\text { Escher-Wyss } \\
\text { Voith }\end{array}$ & A.E.G. & 1964 \\
\hline & Erzhausen & $4 \mathrm{FH}$ & 295,5 & 250 & 296,7 & 75 & 230 & 428 & $\begin{array}{c}\text { Escher-Wyss } \\
\text { Voith }\end{array}$ & Siemens - A.E.G. & 1965 \\
\hline & Schwarzenbach & $2 \mathrm{PH}$ & 340 & 45 & 230 & $3,2 / 4,8$ & & 500 & $\begin{array}{c}\text { Escher-Wyss } \\
\text { Vo:th }\end{array}$ & Siemens - B.B.C. & 1925 \\
\hline \multirow{4}{*}{$\begin{array}{l}\text { magne } \\
\text { rientale }\end{array}$} & Hohenwarte 1 & $2 F$ & 66 & 42 & 67 & 70 & 34 & 166 & & & 1958 \\
\hline & Hohenwarte II & $8 \mathrm{~F}$ & 305 & 320 & 300 & 95,2 & 320 & 428 & C.K.D. Biansko & C.K.D. Biansko & 1965 \\
\hline & Niederwertha & $6 \mathrm{~F}$ & 143 & 129 & 148 & 72 & 120 & 375 & & & 1960 \\
\hline & Wendefurt & $2 F$ & 124 & 80 & 124 & 47 & 70 & 250 & C.K.D. Biansko & C.K.D. Biansko & 1968 \\
\hline
\end{tabular}




\begin{tabular}{|c|c|c|c|c|c|c|c|c|c|c|c|}
\hline \multirow[b]{2}{*}{ Pays } & \multirow[b]{2}{*}{ Station } & \multirow[b]{2}{*}{$\begin{array}{l}\text { Nombre } \\
\text { de groupes } \\
\text { et types }\end{array}$} & \multicolumn{2}{|c|}{ Turbine } & \multicolumn{3}{|c|}{ Pompe } & \multirow[b]{2}{*}{$\begin{array}{l}\text { Vitesse } \\
(t r / m n) \\
\end{array}$} & \multicolumn{2}{|c|}{ Constructeurs } & \multirow[b]{2}{*}{$\begin{array}{c}\text { Année } \\
\text { de mise } \\
\text { en } \\
\text { service }\end{array}$} \\
\hline & & & $\begin{array}{c}\begin{array}{c}\text { Hauteur } \\
\text { de chute } \\
\text { nette }\end{array} \\
(\mathrm{m})\end{array}$ & 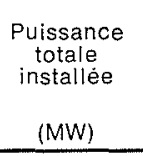 & $\begin{array}{c}\text { Hauteur } \\
\text { totale } \\
\text { d'éléva- } \\
\text { tion } \\
(m) \\
\end{array}$ & $\begin{array}{l}\text { Debit } \\
\text { total } \\
\left(\mathrm{m}^{3} / \mathrm{s}\right) \\
\end{array}$ & $\begin{array}{c}\text { Puissance } \\
\text { totale } \\
\text { absorbée } \\
\text { (MW) }\end{array}$ & & $\begin{array}{l}\text { Turbine } \\
\text { et pompe }\end{array}$ & $\begin{array}{l}\text { Alternateur } \\
\text { moteur }\end{array}$ & \\
\hline \multirow[t]{2}{*}{ Australie } & Tumut 3 & 3 & 151 & 1500 & 156 & 300 & 540 & 187 & Toshiba & Mitsubishi & 1974 \\
\hline & Kangaro Valley & 2 & 440 & 160 & 495 & 28,5 & 320 & 500 & & & 1976 \\
\hline \multirow[t]{17}{*}{ Autriche } & Ranna & $\begin{array}{l}1 \mathrm{FH} \\
2 \mathrm{FH}\end{array}$ & 166,5 & $\begin{array}{c}13,2 \\
4\end{array}$ & & & & & & & 1952 \\
\hline & & 1 Ppe & & & & 6 & 13,4 & & & & \\
\hline & Rodund 1 & $4 \mathrm{FH}$ & 328 & 173 & & & & & & & 1952 \\
\hline & Rodund II & $\begin{array}{l}1 \text { Ppe } \\
\text { voir grol }\end{array}$ & upes réve & rsibles & 345 & $\begin{array}{r}10 \\
(10)\end{array}$ & $\begin{array}{r}40 \\
(40)\end{array}$ & & & & \\
\hline & Limberg & $2 \mathrm{FH}$ & 364 & 112 & & & & & & & 1955 \\
\hline & & 2 Ppe & & & 420 & 33,2 & 130 & & & & \\
\hline & Ottenstein & $4 \mathrm{FV}$ & 59 & 42 & & & & & & & 1957 \\
\hline & & $2 \mathrm{Ppe}$ & & & 62,5 & 34,6 & 10 & & & & \\
\hline & Lünersee & $6 \mathrm{PV}$ & 894 & 263 & & & & & & & 1958 \\
\hline & & 6 Ppe & & & 956 & 28 & 260 & & & & \\
\hline & $\begin{array}{l}\text { Reisseck } \\
\text { Hattelberg }\end{array}$ & $3 \mathrm{PH}$ & 1772 & 60 & 1070 & 1,35 & 19,5 & & & & 1957 \\
\hline & Freibach & $1 \mathrm{FV}$ & 320 & 16,4 & 334 & 1,25 & 5 & & & & 1958 \\
\hline & Haselstein & $1 \mathrm{PH}$ & 279 & 4 & 282 & 1,72 & 5,01 & 993 & Voith & Siemens & 1967 \\
\hline & Oschenik & $2 \mathrm{PH}(+1)$ & 1177 & $66(+33)$ & & & & & & & 1967 \\
\hline & & Ppe & & & $974 / 718$ & $7,6+4,3$ & $57+28$ & 750 & Andrizt & Elin & \\
\hline & Rösshag & $4 \mathrm{FV}$ & 672 & & 607 & & & & & & $?$ \\
\hline & & $2 \mathrm{Ppe}+2$ & & 230 & 607 & $18+18$ & $120+120$ & 750 & Andrizt & Elin & \\
\hline \multirow[t]{3}{*}{ Bulgarie } & Belmeken & $1 \mathrm{~F}$ & 700 & 75 & 700 & 12,5 & 56 & 500 & C.K.D. Biansko & Skoda & 1972 \\
\hline & Antonivanovci & $1 \mathrm{~F}$ & 122 & 45 & 122 & 40 & 48 & 214 & C.K.D. Biansko & Skoda & 1973 \\
\hline & Vitocha & 3 & 897 & 500 & 930 & 41,2 & 420 & & & & projet \\
\hline \multirow[t]{4}{*}{ Espagne } & Puento Bibey & $\begin{array}{l}4 \mathrm{FV} \\
1 \mathrm{Ppe}\end{array}$ & 365,6 & 400 & 361 & 16 & 102 & & Neyrpic & & 1967 \\
\hline & Pintado & $3 \mathrm{FV}$ & 197,5 & 20,6 & 166 & 6 & 14 & & & & 1965 \\
\hline & & 1 Ppe & & & & & & & & & \\
\hline & Ibon de Ip & $3 P$ & 980 & 103,5 & 985 & & 99 & 1000 & Riva & & 1969 \\
\hline \multirow[t]{10}{*}{ France } & Belleville & $2 \mathrm{PH}$ & 530 & & 500 & 0,88 & 6 & & & & 1925 \\
\hline & & 1 Ppe & & & 500 & 1 & 7,3 & & & & \\
\hline & Lassoula & $3 \mathrm{PH}$ & 474 & & 474 & 1,8 & 10 & & & & 1932 \\
\hline & Lac Noir & $4 \mathrm{FV}$ & 126,5 & 100 & 90 & 56,25 & 74,2 & 273 & Escher-Wyss & Alsthom & 1938 \\
\hline & Hospitalet & $3 \mathrm{PH}$ & 730 & 88,5 & & & & 750 & Jeumont & C.E.M. & 1960 \\
\hline & & $1 \mathrm{PH}$ & 535 & 13,8 & & & & 750 & Riva & & \\
\hline & & $2 \mathrm{Ppe}$ & & & 260 & 8 & 23,64 & 750 & Riva & & \\
\hline & Emosson & $3 \mathrm{PV}$ & 803 & 192 & & & & 600 & Alsthom-Neyrpic & Jeumont & 1972 \\
\hline & & $1 \mathrm{FV}$ & 388 & 50,5 & & & & 600 & Charmilles & Schneider & \\
\hline & & 2 Ppe & & & 390 & 18 & 76,4 & 600 & Sulzer & & \\
\hline \multirow[t]{2}{*}{ Hongrie } & Predikaloszek I & $3 F$ & 507 & 307,5 & 515 & 39 & 222,6 & 500 & & & 1978 \\
\hline & Predikaloszek II & $6 F$ & 507 & 615 & 515 & 78 & 446,2 & & & & projet \\
\hline \multirow[t]{12}{*}{ Italie } & Provvidenza & $\begin{array}{l}2 \mathrm{FV} \\
2 \mathrm{Ppe}\end{array}$ & 286 & 150 & 286 & 42 & 140 & 500 & $\begin{array}{c}\text { Asgen } \\
\text { Tosi }\end{array}$ & Marelli & 1950 \\
\hline & Gualdalami & $3 \mathrm{FH}$ & 182 & 80 & 182 & 26 & 50 & 428 & Riva & Breda & 1951 \\
\hline & & $2 \mathrm{Ppe}$ & & & & & & & & & \\
\hline & Villa Garonano & $2 \mathrm{FV}$ & 417 & 134 & 417 & 28 & 134 & 600 & Escher-Wyss & C.G.E. & 1964 \\
\hline & Lete-Sava & $2 \mathrm{PV}$ & 647,1 & 110 & 660 & 14,1 & 105 & 500 & Riva & Ocrem & 1963 \\
\hline & Lago-Delio & $8 \mathrm{H}$ & 753,5 & 1040 & 753,5 & 93,4 & 720 & 500 & $\begin{array}{l}\text { Tosi - Riva } \\
\text { Asgen }\end{array}$ & $\begin{array}{c}\text { Asgen } \\
\text { Tibb Ocrem }\end{array}$ & 1972 \\
\hline & St Florano II & 2 & 1403 & 280 & 1438 & 13,8 & 212 & 600 & Tosi - Asgen & Marelli & 1972 \\
\hline & Fadalto & 2 & 107 & 240 & 116 & 145 & 152 & 176 & Riva & Breda & 1972 \\
\hline & Chiotas-Piastra & 4 & 990 & 540 & 1066 & 46 & 520 & $500 / 600$ & & & 1974 \\
\hline & Rovina Piastra & 1 & 540 & 105 & 588 & 11,1 & 72 & 600 & & & 1974 \\
\hline & Taloro & & 280 & 240 & 290 & & 240 & & & & projet \\
\hline & Piani di Ruschio & & 560 & 480 & 580 & & 400 & & & & projet \\
\hline
\end{tabular}


LA HOUILLE BLANCHE / No 6-7/1972

\begin{tabular}{|c|c|c|c|c|c|c|c|c|c|c|c|}
\hline \multirow[b]{2}{*}{ Pays } & \multirow[b]{2}{*}{ Station } & \multirow[b]{2}{*}{$\begin{array}{l}\text { Nombre } \\
\text { de groupes } \\
\text { et types }\end{array}$} & \multicolumn{2}{|c|}{ Turbine } & \multicolumn{3}{|c|}{ Pompe } & \multirow[b]{2}{*}{$\begin{array}{l}\text { Vitesse } \\
\text { (tr/mn) }\end{array}$} & \multicolumn{2}{|c|}{ Constructeurs } & \multirow[b]{2}{*}{$\begin{array}{c}\text { Annèe } \\
\text { de mise } \\
\text { en } \\
\text { service }\end{array}$} \\
\hline & & & $\begin{array}{c}\text { Hauteur } \\
\text { de chute } \\
\text { nette }\end{array}$ & $\begin{array}{c}\text { Puissance } \\
\text { totale } \\
\text { installée } \\
\text { (MW) }\end{array}$ & $\begin{array}{l}\text { Hauteur } \\
\text { totale } \\
\text { d'éléva- } \\
\text { tion } \\
(\mathrm{m})\end{array}$ & $\begin{array}{l}\text { Débit } \\
\text { total } \\
\left(\mathrm{m}^{3} / \mathrm{s}\right)\end{array}$ & $\begin{array}{c}\text { Puissance } \\
\text { totale } \\
\text { absorbee } \\
\text { (MW) }\end{array}$ & & $\begin{array}{l}\text { Turbine } \\
\text { et pompe }\end{array}$ & $\begin{array}{l}\text { Alternateur } \\
\text { moteur }\end{array}$ & \\
\hline \multirow[t]{2}{*}{ Japon } & Numazawanuma & $2 \mathrm{FH}$ & 215,5 & 46 & 226,2 & 17,7 & 42 & 500 & Hitachi & Hitachi & 1952 \\
\hline & Morozuka & $1 \mathrm{FV}$ & 243,3 & 53 & 245,9 & 18,5 & 49 & 300 & Hitachi & Hitachi & 1960 \\
\hline \multirow[t]{2}{*}{ Luxembourg } & & & & 900 & 290 & 189 & 621 & 428 & $\begin{array}{l}\text { E.W. - Voith } \\
\text { Neyrpic }\end{array}$ & $\begin{array}{l}\text { A.C.E.C. } \\
\text { Siemens }\end{array}$ & 1964 \\
\hline & Vianden & \multicolumn{3}{|c|}{ voir groupes réversibles } & & & & & & & \\
\hline \multirow[t]{2}{*}{ Norvège } & Brattingloss & $1 \mathrm{FH}$ & 122 & 15 & 120 & 7,5 & 12 & 428 & Voith & Siemens & 1953 \\
\hline & Herva & 1 & 245 & 36 & 265 & 10,1 & 36 & 500 & Riva & E.G.A. & 1962 \\
\hline Portugal & Alto Rabagac & 2 & 170 & 36 & 149,5 & 18,5 & 45 & 428 & Vevey & Sécheron & 1960 \\
\hline \multirow[t]{3}{*}{ Roumanie } & Lotru & 7 & 809 & 500 & & & & & & & \\
\hline & Timis & 3 & 726 & & & & & & & & \\
\hline & Vidraru & 2 & 247 & 100 & & & & & & & \\
\hline \multirow[t]{2}{*}{ Royaume Uni } & Flestiniog & $4 \mathrm{FV}$ & 310,9 & 360 & 326,1 & 60 & 73,5 & 428 & English Elec. & A.E.I. & 1963 \\
\hline & Sron Mor & $1 \mathrm{FH}$ & 44,2 & 5 & 44,2 & 11,3 & 5,1 & & & & 1957 \\
\hline Suède & Lotten & $2 \mathrm{FH}$ & 180 & 40,6 & 192,5 & 17,8 & 37,2 & 500 & Maier & A.S.E.A. & 1956 \\
\hline \multirow[t]{2}{*}{ Suisse } & Ferrera I & $\begin{array}{l}3 \mathrm{FH} \\
2 \mathrm{Ppe}\end{array}$ & 522 & 216 & 466 & 10,4 & 40 & 750 & Escher-Wyss & Charmilles & $?$ \\
\hline & Veytaux (Hongrin) & $4 \mathrm{PH}$ & 883 & 240 & 883 & 100 & 240 & 600 & Vevey & $\begin{array}{l}\text { Brown Boveri } \\
\text { Sécheron }\end{array}$ & 1975 \\
\hline Tchécoslovaquie & Dobsina & $2 \mathrm{FH}$ & 265 & 22 & 265 & 5,6 & 17 & & & & 1953 \\
\hline U.R.S.S. & Zagorsk & 2 & 91 & 585 & & & 585 & & L.M.Z. & & $?$ \\
\hline Yougoslavie & Lisina & 2 & & 33 & 363 & 7,5 & 33 & 360 & & & 1973 \\
\hline
\end{tabular}




\section{GROUPES RÉVERSIBLES (caractéristiques unitaires)}
Abréviations
F : Francis
P : Pelton
D : Deriaz
$K$ : Kaplan
$V$ : Vertical
$H$; Horizontal

\begin{tabular}{|c|c|c|c|c|c|c|c|c|c|c|c|}
\hline \multirow{3}{*}{ Pays } & \multirow{3}{*}{ Station } & \multirow{3}{*}{$\begin{array}{l}\text { Nombre } \\
\text { de groupes } \\
\text { et types }\end{array}$} & \multicolumn{5}{|c|}{$\begin{array}{l}\text { Points de fonctionnement } \\
\text { à puissance maximale unitaire }\end{array}$} & \multirow{3}{*}{$\begin{array}{l}\text { Vitesse } \\
(t r / m n)\end{array}$} & \multicolumn{2}{|c|}{ Constructeurs } & \multirow{3}{*}{$\begin{array}{l}\text { Année } \\
\text { de mise } \\
\text { en } \\
\text { service }\end{array}$} \\
\hline & & & \multicolumn{2}{|c|}{ Turbinage } & \multicolumn{3}{|c|}{ Pompage } & & \multirow{2}{*}{\multicolumn{2}{|c|}{$\begin{array}{l}\text { Pompe } \\
\text { turbine }\end{array}$}} & \\
\hline & & & $\begin{array}{l}\text { Hauteur } \\
\text { de chute } \\
\text { nette } \\
\text { (m) }\end{array}$ & $\begin{array}{l}\text { Puissance } \\
\text { unitaire } \\
\text { fournie } \\
\text { (MW) }\end{array}$ & $\begin{array}{c}\text { Hauteur } \\
\text { totale } \\
\text { d'éléva- } \\
\text { tion } \\
(\mathrm{m})\end{array}$ & $\begin{array}{c}\begin{array}{c}\text { Débit } \\
\text { unitaire }\end{array} \\
\left(\mathrm{m}^{3} / \mathrm{s}\right)\end{array}$ & $\begin{array}{c}\text { Puissance } \\
\text { unitaire } \\
\text { absorbée } \\
\text { (MW) }\end{array}$ & & & & \\
\hline $\begin{array}{c}\text { Allemagne } \\
\text { fédérale }\end{array}$ & $\begin{array}{l}\text { Forbach } \\
\text { Rönkhausen } \\
\text { Strittmatt }\end{array}$ & $\begin{array}{l}4 \mathrm{FV} \\
1 \mathrm{FV} \\
4 \mathrm{~F}\end{array}$ & $\begin{array}{l}344 \\
272,6 \\
220\end{array}$ & $\begin{array}{l}100 \\
74,8 \\
36\end{array}$ & $\begin{array}{l}253,4 \\
222\end{array}$ & $\begin{array}{l}23,8 \\
13\end{array}$ & $\begin{array}{l}66,2 \\
32,5\end{array}$ & 500 & EW-Sulzer & Siemens-AEG & $\begin{array}{l}\text { projet } \\
1968\end{array}$ \\
\hline $\begin{array}{c}\text { Allemagne } \\
\text { orientale }\end{array}$ & Markersbach & $6 \mathrm{FV}$ & 301 & 175 & 269,5 & 62 & 177,5 & 375 & & & \\
\hline \multirow{9}{*}{ Autriche } & Antersbach & $4 \mathrm{FV}$ & 150 & 40 & & 26,5 & 47 & 428 & & & projet \\
\hline & Breitenau & $4 \mathrm{FV}$ & 130 & 90 & & 76 & 104 & 250 & & & projet \\
\hline & Inneríragant & $1 \mathrm{H}$ & 268 & 4,1 & 261 & 1,72 & 5,01 & 993 & Voith & & 1968 \\
\hline & Maltatal & $6 \mathrm{~V}$ & 200 & & & 8,9 & 15 & 750 & & & projet \\
\hline & Rield & 4 & 320 & 90 & & 31 & 70 & 750 & & & projet \\
\hline & Rifa & $2 \mathrm{DV}$ & 35 & 4,5 & 29 & 14 & & 300 & Sulzer & & 1968 \\
\hline & Rodund II & $1 \mathrm{FV}$ & 340 & 255 & & 62 & 245 & 375 & & & projet \\
\hline & Sellrainsilz & 2 & 400 & 50 & & 15 & 65 & & & & projet \\
\hline & Weissenkirchen & 4 & 414 & & 334 & 20 & 87,5 & & & & projet \\
\hline Australie & Bendeela & $2 F V$ & 122 & 40 & 133 & 28,5 & 44 & & & & $1976 ?$ \\
\hline Belgique & Coo Trois Ponts & $3+3 F V$ & 269 & 142 & 241 & 55 & 142,3 & 300 & Al.-Chalmers & ACEC & 1970 \\
\hline \multirow[t]{8}{*}{ Brésil } & Edward de Souza & & 26,7 & 11,7 & 23,4 & 51 & 11,7 & 150 & & & 1954 \\
\hline & Pedreira A & $1 \mathrm{KV}$ & 24,7 & 5,3 & 15 & 19,5 & 6 & 212 & Voith & & 1939 \\
\hline & Pedreira $\mathrm{B}$ & $1 \mathrm{KV}$ & 28 & 12 & 28 & 42,5 & 13,5 & 138,5 & Al.-Chalmers & & 1947 \\
\hline & Pedreira $\mathrm{C}$ & 3 & 27,1 & 14,2 & 23,8 & 51 & 14,3 & 150 & Al.-Chalmers & & 1953 \\
\hline & Primavera & 4 & 123 & 123 & 110 & & 125 & 150 & & & projet \\
\hline & Santa Cecillia & 4 & 13,7 & 5,2 & & 40 & 6,5 & 167 & Al.-Chalmers & & 1952 \\
\hline & Traicao & $3 \mathrm{KV}$ & 7 & 2,6 & 7 & 50 & 4,9 & 150 & S. Morgan Smith & & 1939 \\
\hline & Vigario & $4 \mathrm{FV}$ & 29 & 10,8 & 30 & 40 & 13,2 & 150 & Al.-Chalmers & & 1952 \\
\hline \multirow[t]{3}{*}{ Canada } & Brazeau & $2 \mathrm{KV}$ & 13,7 & 12,1 & 13,7 & & 12 & 150 & Dominion & & 1964 \\
\hline & Saint Joachim & 3 & 356 & 406 & & 95,3 & 375 & 257 & & & projet \\
\hline & Sir Adam Beck & $8 \mathrm{DV}$ & 26 & 35,4 & 18,3 & 142 & $\$ 3,2$ & 93,3 & John Inglis & Westinghouse & 1957 \\
\hline \multirow[t]{4}{*}{ Colombie } & Muna II & $1 \mathrm{FV}$ & 31 & 2,43 & 26,8 & 8,7 & 2,87 & 400 & \multicolumn{2}{|l|}{ Ebara (Al. Cs.) } & 1906 \\
\hline & Muna III & $1 \mathrm{FV}$ & 31 & 4 & & & 4 & 400 & \multirow{3}{*}{ Sulzer } & & projet \\
\hline & Sesquille 1 & $1 \mathrm{DV}$ & 37,1 & 3,5 & 32 & 8 & 4,2 & 450 & & & 1964 \\
\hline & Sesquille $\|$ & 2 & 37,1 & 3,5 & 32 & 8 & 4,2 & 450 & & & projet \\
\hline \multirow[t]{8}{*}{ Espagne } & Altamira & $4 \mathrm{FV}$ & 238 & 52 & 262 & 18,35 & 51,7 & 600 & Esch. Wyss & & $1975 ?$ \\
\hline & Bolarque & $4 \mathrm{FV}$ & 258,1 & 56,65 & & & 52,2 & 600 & E.W.-E.E. & & $1975 ?$ \\
\hline & Guillena & $3 \mathrm{FV}$ & 230 & 72 & 220 & 30,4 & 76 & 375 & K.M.W. & & $1974 ?$ \\
\hline & Santiago del Jares & $2 \mathrm{FV}$ & 216,5 & 23,1 & & 9 & & 500 & K.M.W. & Brown Boveri & 1968 \\
\hline & Tajo Segura & 4 & 250 & 55 & & 19 & 57 & & & & projet \\
\hline & Torrejon & $4 \mathrm{FV}$ & 47,7 & 33,1 & 10 & 99 & & 107 & N.B. & Engl. Electric & 1967 \\
\hline & Val de Canas & $3 \mathrm{DV}$ & 74,1 & 84,5 & 72,5 & 104 & 84,5 & 150 & E.E. & & 1964 \\
\hline & Villarino & $4 \mathrm{FV}$ & 392 & 143,5 & 344 & 36,9 & 140 & 600 & K.M.W. & & 1969 \\
\hline
\end{tabular}


a puissance maximale unitaire

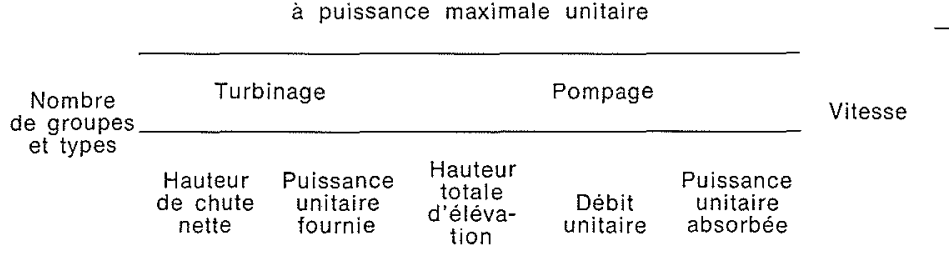

(m) (MW)

(m)

$\left(\mathrm{m}^{3} / \mathrm{s}\right)$

(MW)

(tr/mn)

Bear Swamp

Blair Mountain

Bleinheim Gilboa

Blue Ridge

Breakneck

Cabin Creek

Carters Dam

Castaic Reservoir

Clarence Cannon

Cornwall

Deerfield

De Gray

Dirty Face Mountain

Elbert 1

Flatiron

Forebay

Grand Coulee

Havasu

Hiwassee

Horse Mesa

Jocassee 1

Jocassee II

Kaysinger Bluff

Kinsua

Laurens Shoals

Lewiston

Longwood Valley

Ludington

Merill Lake

Montezuma

Mormon Flat

Muddy Run

Northfield Mountain

Oroville

Racoon Mountain

Salina

San Luis

Semator Wash

Seneca

Smith Mountain

Stony Creek

Taum Sauk

Thermalito

Tocks Island

Tuscarora

Yards Creek
$2 \quad 228,6 \quad 320$

$3 \quad 671 \quad 175$

$4 \mathrm{FV} \quad 339 \quad 300$

$8 \quad 80 \quad 200$

$3 \quad 193 \quad 202$

$2 \mathrm{FV} \quad 362,7$

2 FV 122

328

$8 \mathrm{FV}$

$1 \mathrm{FV}$

I FV

$+\mathrm{FV}$

$1 \mathrm{FV}$

6 DV

$4 \mathrm{FV}$

4

$1 \mathrm{FV}$

$1 \mathrm{FV}$

$4 \mathrm{FV}$

6

2

$4 V \quad 515$

$12 \mathrm{~V} \quad 29$

$6 \mathrm{FV}$

$4 \mathrm{FV}$

1

$8 \mathrm{FV}$

$4 \mathrm{FV}$

$3 \mathrm{FV}$

$4 \mathrm{FV}$

3

$8 \mathrm{FV}$

2

$2 \mathrm{FV}$

6

$3 \mathrm{FV}$

$5 \quad 360$

$12 \mathrm{FV} \quad 29$

$3 \mathrm{FV} \quad 230$

\section{6,2}

125

261

32

256

610

$52,1 \quad 33,2$

$205 \quad 65$

$135 \quad 100$

88,4

110

305
58

58
79

97,5

2300

300
26,6

198

125

45

45
343

343

250

125

41,3

125

244

188

512
71,6

390

46

7,2

168

325

225

260

26
$112,6 \quad 223$

106

31

135

89

43,5

107

226

152

286

60

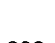

330

$\begin{array}{lll}81 & 319 & 257 \\ 227 & 200 & 112\end{array}$

$\begin{array}{lll}100 & 210 & 150\end{array}$

90,8

$22,9-128$

45,8

73,2

17,1

52,5

89,6

154

$$
71,5
$$

198

$21,25 \quad 125$

25,9

96,4

44

314
61

$496 \quad 23,4$

74,7

102

96115

$93 \quad 217$

217

386

119

65,7

52,6

$$
52,7
$$

$90,8 \quad 195$

89

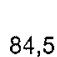

84,5

59
96

41
Constructeurs

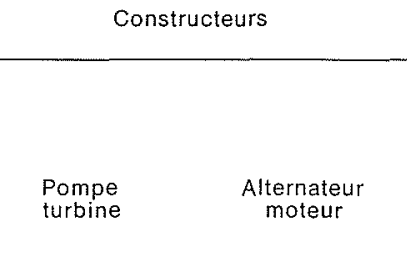

Annèe de mise

en
service 


\begin{tabular}{|c|c|c|c|c|c|c|c|c|c|c|c|}
\hline \multirow{3}{*}{ Pays } & \multirow{3}{*}{ Station } & \multirow{3}{*}{$\begin{array}{l}\text { Nombre } \\
\text { de groupes } \\
\text { et types }\end{array}$} & \multicolumn{5}{|c|}{$\begin{array}{l}\text { Points de fonctionnement } \\
\text { a puissance maximale unitaire }\end{array}$} & \multirow{3}{*}{ Vitesse } & \multicolumn{2}{|c|}{ Constructeurs } & \multirow{3}{*}{$\begin{array}{c}\text { Année } \\
\text { de mise } \\
\text { en } \\
\text { service }\end{array}$} \\
\hline & & & \multicolumn{2}{|c|}{ Turbinage } & \multicolumn{3}{|c|}{ Pompage } & & \multirow[b]{2}{*}{$\begin{array}{l}\text { Pompe } \\
\text { turbine }\end{array}$} & \multirow[b]{2}{*}{$\begin{array}{l}\text { Alternateur } \\
\text { moteur }\end{array}$} & \\
\hline & & & $\begin{array}{c}\begin{array}{c}\text { Hauteur } \\
\text { de chute } \\
\text { nette }\end{array} \\
\text { (m) }\end{array}$ & $\begin{array}{c}\text { Puissance } \\
\text { unitaire } \\
\text { fournie } \\
\text { (MW) } \\
\end{array}$ & $\begin{array}{c}\text { Hauteur } \\
\text { totale } \\
\text { d'éléva- } \\
\text { tion } \\
(m) \\
\end{array}$ & $\begin{array}{c}\begin{array}{c}\text { Débit } \\
\text { unitaire }\end{array} \\
\left(\mathrm{m}^{3} / \mathrm{s}\right) \\
\end{array}$ & $\begin{array}{c}\text { Puissance } \\
\text { unitaire } \\
\text { absorbée } \\
\text { (MW) }\end{array}$ & & & & \\
\hline \multirow[t]{7}{*}{ France } & Arc Isère & $1 \mathrm{FV}$ & 243 & 240 & 258 & 82,5 & & 250 & & & projet \\
\hline & La Coche & $2 F V+2$ & 930,67 & 79 & 882,99 & 8,29 & 80,64 & 600 & Vevey & & $1976 ?$ \\
\hline & Montezic & $4 \mathrm{FV}$ & 410 & 187,5 & & & 155 & & & & projet \\
\hline & Rance & $24 \mathrm{KH}$ & 11 & 10 & 3 & 170,5 & 10 & 93,75 & $\begin{array}{l}\text { Consortium } \\
\text { français }\end{array}$ & & 1968 \\
\hline & Revin & $4 \mathrm{FV}$ & 243 & 197,9 & 234 & 64 & 163,7 & 300 & N.B.-S.F.A.C. & $\begin{array}{l}\text { Alsth. Jeum. } \\
\text { Schneider }\end{array}$ & $1974 ?$ \\
\hline & Sainte Croix & $1 \mathrm{FV}$ & 76,87 & 58,66 & 63,88 & 70,6 & 48,8 & 167 & $\begin{array}{l}\text { Als.-Creus. } \\
\text { Loire }\end{array}$ & C.E.M. & $1975 ?$ \\
\hline & Vouglans & $1 \mathrm{FV}$ & 100,2 & 62 & 89,53 & 61,2 & 59,9 & 150 & C.A.F.L. & Alsthom & $1973 ?$ \\
\hline Irlande & Turlough'Hill & $4 \mathrm{FV}$ & 299 & 78 & 288 & 22,1 & 70 & 500 & K.M.W. & Siemens & $?$ \\
\hline \multirow[t]{2}{*}{ Italie } & Brasimone Soviana & $2 \mathrm{FV}$ & 378 & 169 & & 37 & 150 & 375 & $\begin{array}{c}\text { Riva } \\
\text { Escher Wyss }\end{array}$ & & $1973 ?$ \\
\hline & Provvidenza & $1 \mathrm{FV}$ & 274 & & 262 & 17 & & 375 & Al. Cs. & Asgen Marelli & 1962 \\
\hline \multirow[t]{29}{*}{ Japon } & Agehara & 4 & 459,6 & 272 & & 53,5 & & 360 & & & 1970 \\
\hline & Ananaigawa & $1 \mathrm{DV}$ & 69,5 & 13,5 & 40 & 14,6 & & 360 & Hitachi & Hitachi & 1964 \\
\hline & Azumi & $4 \mathrm{FV}$ & 133,7 & 107,8 & 79,4 & 90 & 109 & 188 & Toshiba & & 1969 \\
\hline & Hakusuidaki & 2 & 474,7 & 225 & & 41 & 232 & & & & $1972 ?$ \\
\hline & Hatanagi 1 & $1 \mathrm{FV}$ & 101,8 & 51,8 & 61,9 & 53 & 41,8 & 200 & Al. Cs. & Fuji Hitachi & 1962 \\
\hline & Hatanagi II & $2 \mathrm{FV}$ & 101,8 & 32,4 & 57 & 49,5 & 46,5 & 200 & Hitachi & & 1962 \\
\hline & |kehara I & $2 \mathrm{FV}$ & 129,5 & 80 & 91 & 75 & & 180 & Hitachi & Hitachi & 1965 \\
\hline & Ikehara II & $2 \mathrm{FV}$ & 129,5 & 110 & 95 & 100 & 110 & 150 & Hitachi & & 1967 \\
\hline & Kagedaira & $1 \mathrm{DV}$ & 89,7 & 47,7 & & 40 & 47 & 240 & Hitachi & & 1968 \\
\hline & Kisenyama & $2 \mathrm{FV}$ & 220 & 240 & 197 & 110 & 240 & 225 & $\begin{array}{l}\text { Hitachi } \\
\text { Toshiba }\end{array}$ & $\begin{array}{l}\text { Hitachi } \\
\text { Toshiba }\end{array}$ & 1970 \\
\hline & Kuromatagawa & $1 \mathrm{DV}$ & 78 & 19,2 & 80 & 21,7 & & $\begin{array}{l}\mathrm{T}=300 \\
\mathrm{P}=333\end{array}$ & Fuji Electric & Fuji Electric & 1964 \\
\hline & Masegawa 1 & 2 & 105 & 148 & & 167,5 & 154 & 180 & & & $1972 ?$ \\
\hline & $\begin{array}{l}\text { Midono } \\
\text { Mio }\end{array}$ & $2 \mathrm{FV}$ & 79,8 & 64 & $\begin{array}{l}59,8 \\
\text { (voir }\end{array}$ & $\begin{array}{c}93 \\
\text { Otakigaw }\end{array}$ & (a) 62,4 & 150 & Hitachi & & 1969 \\
\hline & Nagano & $2 \mathrm{FV}$ & 107,5 & 113 & 71 & 136,5 & 113 & 150 & Hitachi & & 1968 \\
\hline & Nikappu & $2 \mathrm{FV}$ & 94 & 105 & & & & 231 & & & 1970 \\
\hline & Numappara & $3 \mathrm{FV}$ & 500 & 244 & & 50 & 260 & 375 & Hitachi & & $1973 ?$ \\
\hline & $\begin{array}{l}\text { Omorigawa } \\
\text { Okutataragi }\end{array}$ & $\begin{array}{l}1 \mathrm{FV} \\
4 \mathrm{FV}\end{array}$ & 118 & $\begin{array}{l}12,2 \\
250\end{array}$ & 92 & 13 & 14,3 & 400 & Hitachi & Hitachi & $\begin{array}{l}1959 \\
1974 ?\end{array}$ \\
\hline & Otakigawa & $1 \mathrm{FV}$ & 130 & 36 & 104 & 31 & 37 & $\begin{array}{l}277 \\
231\end{array}$ & Hitachi & & 1963 \\
\hline & Ohira & $1 \mathrm{FV}$ & 515 & 250 & & & & 400 & & & projet \\
\hline & Seibu Yosul & 4 & 397,6 & & & & & & & & projet \\
\hline & Shin Narihagawa & $3 \mathrm{FV}$ & 94,7 & 78 & 57,2 & 106,3 & & 114 & Hitachi & & 1970 \\
\hline & Shin Takase & 4 & 230 & 330 & & 137 & 330 & 214,3 & & & $1975 ?$ \\
\hline & Shin Toyone & 5 & 230 & 230 & & 82,4 & 233,5 & $\begin{array}{l}214,3 \\
257\end{array}$ & & & $1972 ?$ \\
\hline & Shiroyama I & $2 \mathrm{FV}$ & 181,4 & 65 & 133 & 46,7 & 64,9 & 273 & Toshiba & $\begin{array}{l}\text { Toshiba } \\
\text { Hitachi }\end{array}$ & 1965 \\
\hline & Shiroyama II & $2 \mathrm{FV}$ & 181,4 & 65 & 133 & 44 & 71,8 & 300 & Hitachi & & $?$ \\
\hline & Takane 1 & $2 \mathrm{DV}$ & 136,2 & 87,3 & 137,6 & 61,7 & 97,7 & 277 & $\begin{array}{l}\text { Mitsubishi } \\
\text { Hitachi }\end{array}$ & & 1969 \\
\hline & Takane II & & 136,2 & 87,3 & 137,6 & 66,3 & 99,4 & 277 & & & projet \\
\hline & Yagisawa I & $2 \mathrm{FV}$ & 111 & 87,5 & 63 & 110 & 87 & 150 & Al. Cs. & & 1965 \\
\hline & Yagisawa 11 & $1 \mathrm{FV}$ & 111 & 87,5 & 63 & 110 & 87 & 150 & Hitachi & & 1967 \\
\hline Luxembou & Vianden & $1 \mathrm{FV}$ & & 196 & & 74,1 & 215 & 333 & $\begin{array}{l}\text { Escher-Wyss } \\
\text { Voith }\end{array}$ & $\begin{array}{l}\text { Siemens } \\
\text { Brown Boveri }\end{array}$ & 1972 \\
\hline
\end{tabular}




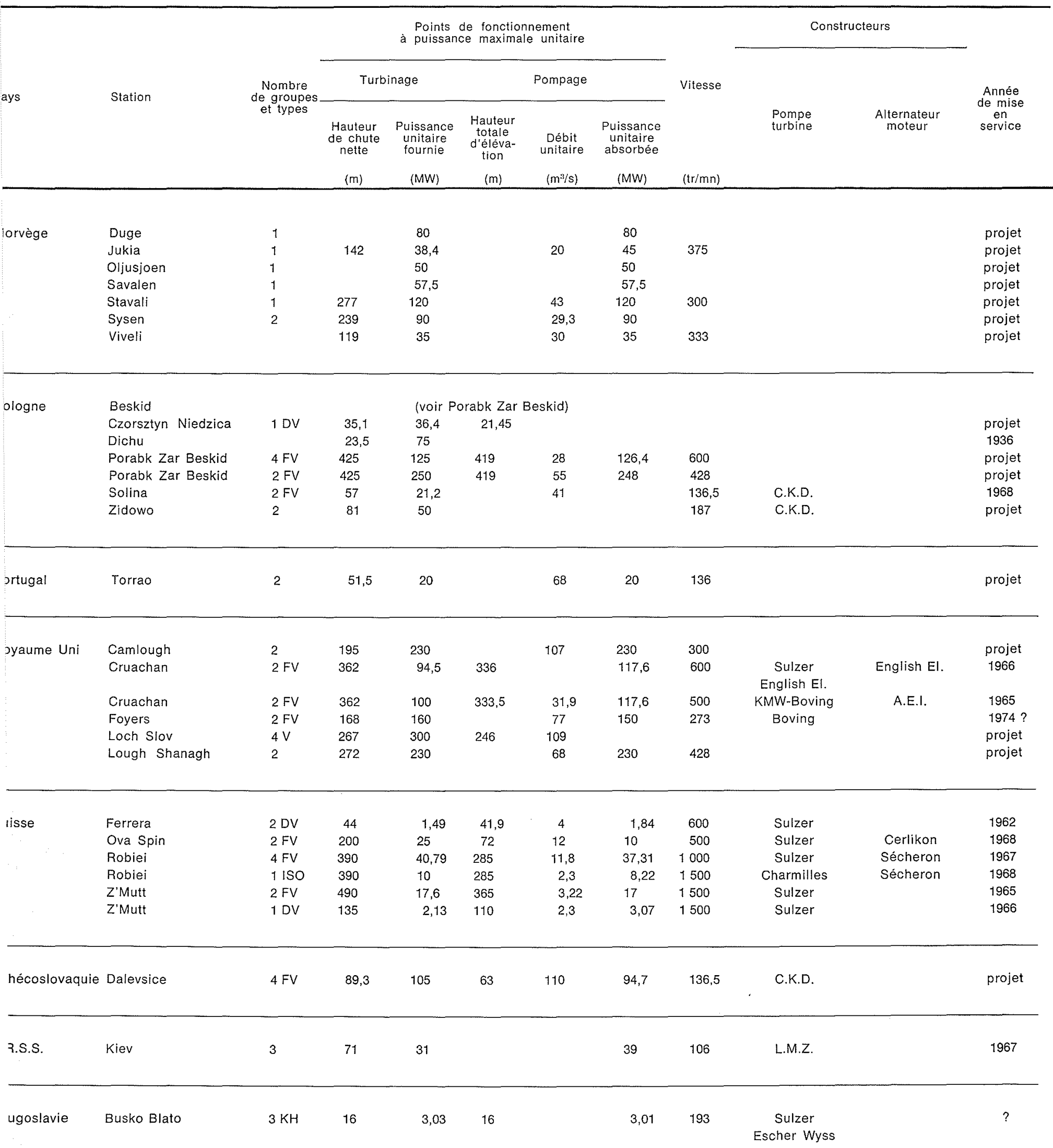

